\title{
Significant Differences in Elderly Health between Urban and Rural Communities: A Literature Review
}

\author{
Junko Imaiso \\ Kansai University of Social Welfare, Ako-shi, Hyogo Prefecture, Japan \\ Email: imaiso@kusw.ac.jp
}

How to cite this paper: Imaiso, J. (2019) Significant Differences in Elderly Health between Urban and Rural Communities: A Literature Review. Health, 11, 567-577. https://doi.org/10.4236/health.2019.115048

Received: April 11, 2019

Accepted: May 28, 2019

Published: May 31, 2019

Copyright (c) 2019 by author(s) and Scientific Research Publishing Inc. This work is licensed under the Creative Commons Attribution-NonCommercial International License (CC BY-NC 4.0). http://creativecommons.org/licenses/by-nc/4.0/

\begin{abstract}
This study aimed to examine measures pertaining to elderly health in urban versus rural settings, and to identify differences in the health of elderly people living in urban and rural communities through a literature review. An electronic literature search was performed using PubMed for English articles published in peer-reviewed journals up to August 2018, with the following search terms: "urban", "rural", "comparison of community", "elderly health", and "comparison of community health". A total of 35 articles were extracted for a critical full-text review, and six articles that met the inclusion criteria were subjected to analysis. Measures related to elderly health in urban and rural communities were classified into the following three categories: functional abilities, health, and health perception. Five of the six articles described functional abilities (e.g., social function) and health (e.g., mental health, depression) as categories with significant differences in elderly health between urban and rural communities. The results suggest that elderly health measures related to social function and mental health or depression are more important outcome measures of effective person-centered integrated community care systems from the perspective of community characteristics. As there were only a few articles reporting on elderly health according to differences in environment between urban and rural communities, further investigation is globally warranted.
\end{abstract}

\section{Keywords}

Aging Society, Elderly Health, Person-Centered Integrated Community Care System, Rural, Urban

\section{Introduction}

Japan is the world's fastest super-aging society. In 2015, elderly people aged 65 
years and older accounted for $26.6 \%$ of the entire population, and this proportion is estimated to increase to $38.0 \%$ in 2055 [1]. As other countries in the world are also facing similar social situations, aging is a global concern. While aging is natural for human beings and does not pose issues itself, the increasing need for care associated with aging is problematic. Konishi [2] suggested that a systematic community strategy would need to be developed in order to improve the quality of life (QOL) of elderly people, while recognizing the importance of living a long, healthy life in their own communities until death. It was also recommended that not only QOL but also the quality of death be considered in elderly care [3] [4]. Thus, there is a need to promote continuous care within the community from the perspectives of prevention, medical care, and caregiving.

In Japan, care needs among elderly people are expected to increase as baby boomers (born between 1947 and 1949) reach the age of 75 years or older in 2025. In order to provide uniform community services pertaining to housing, medical treatment, caregiving, prevention (including primary, secondary, and tertiary), and support in daily life across the country, the Japanese Ministry of Health, Labour and Welfare proposed in 2012 that an integrated community care system be established in each community by 2025. The "Draft Act on Amendatory Law to the Related Acts for Securing Comprehensive Medical and Long-Term Care in the Community" was enacted in 2014, in anticipation of social changes in the near future [1]. The government aims to promote the integration of medical care and long-term care in community caregiving services in a way that is tailored to the characteristics of each community. It is suggested that evidence is lacking with regard to the effectiveness of integrated community care [5]. Constructing an integrated community care system in each community would not bring about significant effects, unless it ensures the effectiveness and sustainability of community care services for the residents themselves. To this end, the sense of person-centered care and a better understanding of each residential community must be cultivated.

Kanagawa [6] suggested that "Community" was diverse. In the field of gerontology, community could be conceptualized or studied as a) something that strongly influences older adults' well-being and QOL, b) an entity that older adults contribute to, or c) a method/methodology in and of itself [7]. Understanding one's community will help perceive the characteristics of the residents of that community, and this, in turn, will lead to the development of an effective, person-centered, integrated community care system.

The characteristics of a community can be defined according to differences in access to health resources, or connections among people in urban versus rural settings. In urban communities, geographical conditions for accessing social support are more convenient, and although the quantity and quality of social support may be higher, the population size is greater, the population density is higher, and relationships within the community tend to be weaker compared to rural communities [8] [9]. On the other hand, in rural communities, human relationships tend to be more intimate, and informal support from relatives or 
neighbors are more prevalent compared to urban communities [8] [10], although formal social support tends to be weaker and resistance against outside help and formal support is more widespread [10]. Furthermore, rural areas have higher population reduction and declining birth rates, larger elderly resident populations, and significantly higher rates of out-migration of younger adults [8] [10]. Thus, there may be some significant differences in elderly health according to differences in environment between urban and rural communities. Thus, it will be important to clarify differences in elderly health between urban and rural communities.

This study aimed to examine measures pertaining to elderly health in urban versus rural settings, and identify differences in elderly health between urban and rural communities through a literature review.

\section{Method}

In the current study, PubMed was used as a research database, because the academic articles related to medical, health and welfare are able to be widely overviewed. The process of the articles search by PubMed was showed (Figure 1).

An electronic literature search was performed using PubMed for English articles published in peer-reviewed journals up to August 2018. The following two combinations of search terms were used: 1) "urban" and "rural" and "comparison of community" and "elderly health"; 2) "urban" and "rural" and "comparison of community health". Identified articles were screened on the basis of title and abstract, and selected articles were subjected to full-text assessment and critical review according to the following inclusion criteria: studies comparing the health of elderly people between urban and rural settings, and those in which participants lived in urban or rural areas and had few disorders. Similar measures of elderly health in urban and rural areas were assessed by collecting corresponding information from each article. These data were then compared in order to identify significant differences in elderly health between the two areas.

\section{Result}

Six articles that met the inclusion criteria were included in the analysis (Figure 1).

In the combination of search terms: 1) "urban" and "rural" and "comparison of community" and "elderly health", 30 articles were identified. In another combination of search terms: 2) "urban" and "rural" and "comparison of community health", five articles were identified. 35 articles were screened. There were no duplicate articles between the 30 articles and the five articles which were identified based on the two combinations of the search key words. Among the 35 identified articles in the electric literature search, 28 articles were excluded after the titles and abstracts were reviewed. Seven articles were selected on the basis of title and abstract and were subjected to a critical review of the full text. One full text article was excluded due to elderly with limited disorders, neurological disorders, as research participants. 


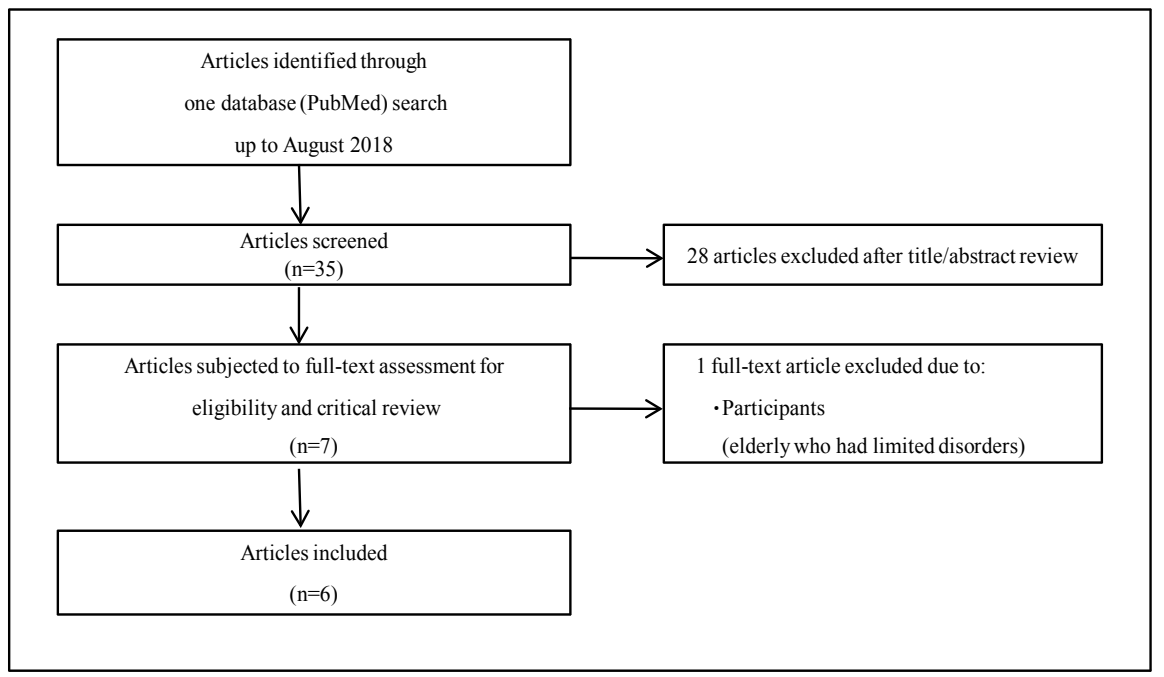

Figure 1. Flowchart of literature search.

Table 1 summarizes data extracted from the six articles. All were reports of quantitative studies conducted in the U.S. $(n=3)$ : [11] [12] [13], Taiwan $(n=2)$ : [14] [15], and Thailand $(n=1)$ : [16]. The four articles [11] [12] [14] [15] were correlation research design with multivariate procedure. The definitions of urban and rural areas in the respective countries were provided in four articles [11] [12] [13] [15]. For instance, "urban" meant the metropolitan statistical area (MSA) or centralized area based on characteristics such as population size or population density, or environmental aspects. Two articles [14] [16] lacked clear definitions for the two types of areas. And then, three articles [12] [13] [15] explained the socio-demographic characteristics of the study in urban and rural such as age, gender, ethnic group, marital status, education, employment, living arrangement, financial, number of relatives feels close to. It was not statistically clear about the difference between urban and rural in one article [13]. The other two articles [12] [15] in common showed the significant different variable between urban and rural; Ethnic group (Race). And then, Friedman et al. [12] showed one significant different variable number of relatives feel close to, between urban and rural $(\mathrm{p}=0.022)$.

Two articles [13] [14] only included female participants. Four articles [11], [12] [14] [15] targeted elderly participants aged 65 years and older, and two articles [13] [16] targeted those aged 60 years and older. The number of participants ranged from 72 ( 39 urban and 33 rural) to 1005 (678 urban and 327 rural). The 10 main instruments used to assess health status in the six articles were as follows: 20-item Short-Form Health Survey (SF-20), Social Class Scale, Perceived Health Scale (PHS), Exercise of Self-Care Agency Scale (ESCAS), Positive Affect Scale (PAS), Geriatric Depression Scale (GDS), Functional Disability in ADL Activities, Health Promoting Lifestyle Profile II (HPLP-II), World Health Organization Quality of Life Questionnaire (WHOQOL-BREF), and General Health Questionnaire (GHQ-28). 
Table 1. Overview of data extracted from analyzed articles.

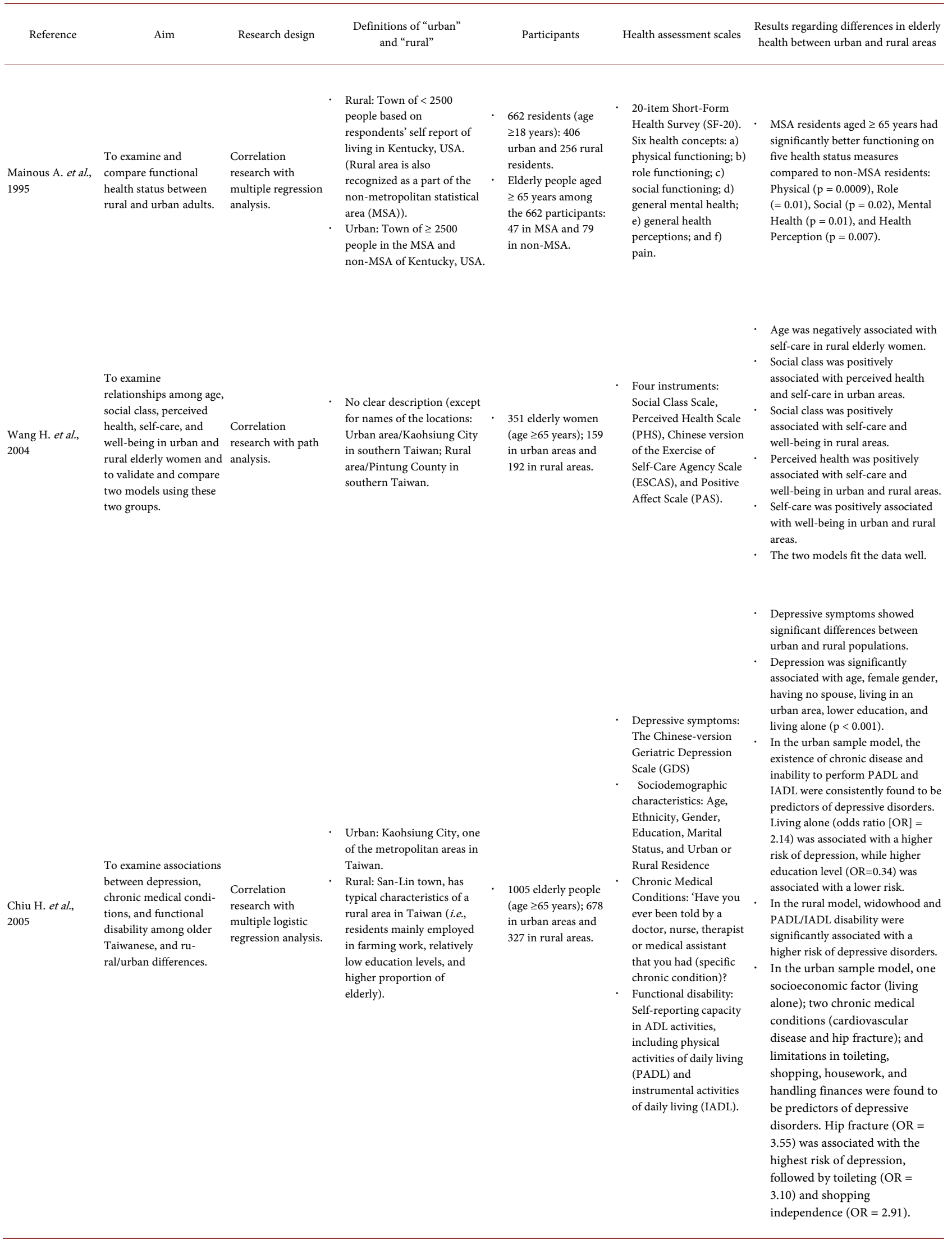




\section{Continued}

To determine whether Cross-sectional

Friedman B. et al., 2007

$\begin{aligned} & \text { To identify and } \\ & \text { compare Health } \text { Promotion Behaviors } \\ & \text { Lee C. et al., } 2009 \text { (HPBs) of older rural } \\ & \text { and urban } \text { community-dwelling } \\ & \text { female spousal } \\ & \text { caregivers. }\end{aligned}$

Exploratory, descriptive survey design. caregivers. factors associated with observational · Urban: MSA

- Urban: MSA
on the U.S. Office of $\begin{array}{lll}\text { between elderly resi- } & \text { multiple logistic } & \text { on the U.S. Office of } \\ \text { dents of rural and } & \text { regression } & \text { Management and Budget) }\end{array}$ urban areas. Management and Budget)
926 elderly people (age $\geq 65$ years); 650 in urban areas and 276 in rural areas. status, number of 13 with a population density of 1000 per square mile and at least 10,000 total.

Rural: Outside the urbanized cluster or areas with rural status designation.
- Sociodemographic gender, education, annual household income, financial strain, and site.

Social support: marital status, living arrangement, number of friends, and number of relatives.

- Supplemental health insurance

Prior health services use

- Health status: self-related health chronic conditions, SF-36 Physical Component Summary (PCS) score, anxiety, body mass index (BMI) number of ADL dependencies, number of IADL dependencies

HPLP-II: 52

health-related behaviors and six subscales; health responsibility, nutrition, physical activity, spiritual

growth, interpersonal - No significant differences relations, and stress management.

- In the rural sample, widowed elders, stroke history, and problems with toileting and shopping were significantly associated with a higher risk of depressive disorders. For rural elderly with a stroke history, there was a 7.39 increase in likelihood of suffering from depression.

Major depression (SF-36 PCS score $>30$ ) significantly differed between urban and rural areas ( $p=0.007)$; urban elderly had major depression significantly more often than rural elderly. For urban elderly, major depression was associated with dependence in five or six ADL (OR: 2.33) and in three or more instrumental ADLs (OR: 8.85).

Having very few close friends (OR: 6.86, 95\% confidence interval [Cl]: 2.18 - 21.58), $2+$ emergency room visits in the past 6 months (OR: 4.00, 95\% $\mathrm{Cl}: 1.19$ - 13.43), and mor financial strain (OR: 1.50, 95\% $\mathrm{Cl} 1.01-2.23)$ were associated with a significantly higher likelihood of major depression in rural areas as compared with urban areas. people (age $\geq 60$ years) who are provided with care for at least 6 months at home; 39 in urban areas and 33 in rural areas.
To assess quality of life and mental and physical Apidechkul T., health among people Cross-sectional 2011 aged aged 60 years and over study. in urban and rural areas of northern Thailand.
(Not written clearly)

Urban: 11 villages in the $\mathrm{Pa}$ Kaw Dum sub-district, and the Mae Lao district were used as suburban areas.
225 elderly people (age $\geq 60$ years); 116 in urban areas and 109 in rural areas.
- PI-developed questionnaire: demographics, caregiving responsibilities, length of time in caregiving role, and amount of personal time.

WHOQOL-BREF-THA I: 26 questions/ physical health, mental health, social relationships, and environment health.
Subjects who lived in suburban areas had a higher quality of life than those who lived in rural areas in terms of physical health $(\mathrm{p}=$ $0.011)$, mental health $(\mathrm{p}=0.025)$, and social relationships $(\mathrm{p}=0.012)$ 
Rural: Seven villages in the

Pa Tung sub-district, and the

Mae Chan district were used

as rural areas.

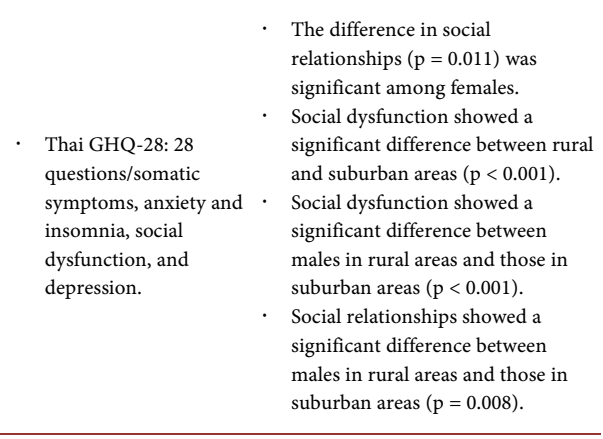

Measures pertaining to elderly health in urban and rural communities described in the six articles were classified into the following three categories: functional abilities, health, and health perception. Five [11] [12] [14] [15] [16] of the six articles noted significant differences in elderly health in terms of functional abilities, health, or health perception between urban and rural communities, whereas one article [13] found no significant difference in the above three categories.

\subsection{Functional Abilities}

Functional abilities included the following three elements: social function, physical function, and role function in daily life. Three articles [11] [14] [16] noted significant differences in social function between urban and rural communities. Mainous et al. [11] reported that urban residents aged $\geq 65$ years had better social functioning compared to their rural counterparts ( $\mathrm{p}=0.02)$, and Wang et al. [14] reported that social class was positively associated with perceived health and self-care in urban areas. Apidechkul [16] reported that social dysfunction significantly differed between rural and suburban areas $(\mathrm{p}<0.001)$, and that social relationships in urban areas were higher than those in rural areas $(p=0.012)$. Moreover, Friedman et al. [12] found that the social aspect (e.g., social relationships, having very few close friends) was a significant factor associated with mental health or depression when comparing urban versus rural communities. For instance, having very few close friends (odds ratio [OR]: 6.86; 95\% confidence interval $[\mathrm{Cl}]: 2.18$ - 21.58) was associated with a significantly higher likelihood of major depression in rural areas compared to urban areas. The other two elements, physical function $(\mathrm{p}=0.0009)$ and role function in daily life $(\mathrm{p}=$ 0.02 ), were reported to be significantly better in urban areas compared to rural areas in one article [11].

\subsection{Health}

The health category included the following two elements: mental health or depression and physical health. Four articles [11] [12] [15] [16] noted significant differences for mental health or depression. Among those, two articles [11] [16] found that mental health or depression in elderly people was significantly better 
in urban areas than in rural areas. The other two articles [12] [15] reported that mental health or depression in elderly people significantly differed between urban and rural areas. Chiu et al. [15] showed significant differences between urban and rural populations $(\mathrm{p}=0.005)$, and depression was significantly associated with living urban area (odds ratio [OR]: 0.59; 95\% confidence interval [Cl]: 0.40 - 0.85). Friedman et al. [12] found a significant difference in the incidence of major depression between urban and rural areas $(\mathrm{p}=0.007)$, with those living in urban areas being significantly more prone to major depression.

Two articles [11] [16] noted significant differences between urban and rural settings for physical health. One article [11] found that physical health differed significantly $(\mathrm{p}=0.0009)$ according to the SF-20 subscale, and another article [16] found that physical health in urban areas was higher compared to rural areas $(\mathrm{p}=0.011)$.

\subsection{Health Perception}

Two articles [11] [14] reported on health perception with a focus on urban and rural settings. Mainous et al. [11] found a significant difference $(\mathrm{p}=0.007)$ between urban and rural communities, and Wang et al. [14] showed a positive association between perceived health and self-care and well-being in urban and rural communities.

\section{Discussion}

The population composition in Japan continues to change with the increasing elderly population and decreasing birth rates. Under such social circumstances, the characteristics of a community will continue to change; like humans, the community is alive.

The Japanese government is currently promoting the construction of integrated community care systems tailored to the characteristics of each residential community by 2025. "Community" is diverse [6], and while it has been suggested to strongly influence older adults' well-being and QOL [7], the definition of "community" in a research setting is still unclear. Therefore, the present study used "urban" and "rural" settings as characteristics of residential communities according to the literature [8] [9] [10]. And then, through a literature review of six studies including the four articles [11] [12] [14] [15] of the correlation research design with multivariate procedure, was carried out, and significant differences identified in elderly health between urban and rural communities were classified into three categories (functional abilities, health, and health perception). Five [11] [12] [14] [15] [16] of the six studies identified significant differences in elderly health relating to functional abilities and health.

Among functional abilities, social function was described in five articles [11] [12] [13] [14] [16]. In one article [16], the social aspect (e.g., social relationships, having very few close friends) was a significant factor associated with mental health or depression when comparing urban and rural areas. In particular, hav- 
ing very few close friends was associated with a significantly higher likelihood of major depression among rural residents compared to urban residents. It is suggested that the important issues might be what the whole community (ie, urban or rural) understands about the life of the people, rather than the views of the actual communities that the people live in [17]. The findings in the current study suggest that social function might be a more important outcome measure of elderly health, particularly regarding mental health or depression, when considering outcome measures for effective integrated community care that takes into account the characteristics of each community.

In the health category, mental health was described in five articles [11] [12] [13] [15] [16]. Among these, four articles [11] [12] [15] [16] reported that mental health or depression in elderly people significantly differed between urban and rural communities. However, the results regarding the mental state of elderly people in urban versus rural communities were not consistent among these articles; two articles [11] [16] reported that urban elderly people had better mental health compared to rural elderly people, whereas other two articles [12] [15] found mental health in rural elderly people to be better. Thus, whether a significant difference exists in elderly mental health between urban and rural communities remains unclear. Yet, mental health or depression might be a more important measure of elderly health in effective integrated community care based on community characteristics.

Overall, the definitions of urban and rural areas in the respective countries seemed not to be enough provided. And then, through a literature review, the present study revealed that significant differences between urban and rural communities relate to aspects of social function and mental health or depression, although only a few studies reported on elderly health from the perspective of differences in environment between urban and rural communities. One article [16] showed that urban elderly people had higher QOL compared to their rural counterparts, in terms of mental health and social function. When developing an effective person-centered integrated community care system that aims to promote the QOL of residents while taking into consideration the characteristics of each community (i.e., urban or rural), it will be important to recognize social aspects as well as mental health or depression as more important outcome measures. In addition to it, on this article, Chiu et al. [15] showed that depression was significantly associated with living urban area. One possibility is that the residential community itself such as urban and rural may be one of the factors influencing the mental health or depression.

In order to carry out further studies regarding effective integrated community elderly care that considers community characteristics in the future, the definition of the characteristics of community (e.g., urban and rural) in research settings should be clarified. And then, further studies will be needed that focus on not only elderly health but also the environmental aspects surrounding community residents (urban/rural) with intervention research design, in order to explore outcome measures for effective person-centered integrated community 
care systems. In addition, it will be more important to pile the research data globally and perform a meta-analysis.

\section{Acknowledgements}

A part of manuscript was accepted as Peer-Reviewed Poster Presentation at the $30^{\text {th }}$ International Nursing Research Congress, Calgary, Alberta, Canada, in Sigma Theta Tau International, 25-29 July, 2019.

\section{Conflicts of Interest}

The authors declare no conflicts of interest regarding the publication of this paper.

\section{References}

[1] Health and Welfare Statistics Association (2018) Kokumin eisei no doukou (Tendency about People's Health). Journal of Health and Welfare Statistics, 65, 49, 187.

[2] Konishi, M. (2015) Koureisha ga anshin shite kurasu kenkou komyunityi wo mezashite (Systematic Strategy of the Community for Improving the Quality of Life of the Elderly). Gakujyutsu no Doukou (Trends in the Sciences), 20, 59.

[3] Lanoë, R.S., Chapiro, S.L., Feteanu, D., and Trivalle, C. (2003) Palliative Care in a Long-Term Care Setting: A 25-Years French Experience. Journal of Palliative Care, 19, 209-213. https://doi.org/10.1177/082585970301900311

[4] Kamata, K. (2015) Koureisha mondai no kakushin to kea no yakuwari (The Core Problem in the Elderly and the Role of Care). Journal of Caregiving of the Elderly, 43, 1-9.

[5] Imaiso, J. (2018) Outcomes of Integrated Community Care Interventions for Frail Elderly People: A Literature Review. Health, 10, 1120-1131. https://doi.org/10.4236/health.2018.108085

[6] Kanagawa, K. (2014) Chiiki kango katsudou ni okeru tokutei hi eiri katsudou no igi- NPO houjin ishikawa zaitaku netto wo tachiagete- (The Significance of the Activities by Nonprofit Organization(NPO) in Community Health Nursing-Starting Home Care Network by NPO Ishikawa-). Journal of Japan Academy of Community Health Nursing, 17, 6-9.

[7] Gaugler, J.E. (2015) Aging of Communities: Communities of Aging. Journal of Applied Gerontology, 34, 135-137. https://doi.org/10.1177/0733464814562271

[8] Glasgow, N. (2000) Rural/Urban Patterns of Aging and Caregiving in the United States. Journal of Family Issues, 21, 611-631. https://doi.org/10.1177/019251300021005005

[9] Japanese Ministry of Health, Labor and Welfare (2005) Kousei roudou hakusho chiiki to tomoni sasaeru korekarano shakai hosho (White Paper in Japanese Ministry of Health, Labor and Welfare Future Social Security to Support with Community). Tokyo, Japan.

[10] Bushy, A. (2000) Nursing and Rural Health Care Delivery. In: Janke, R., Ed., Orientation to Nursing in the Rural Community, Sage, Thousand Oaks, CA, 1-60.

[11] Mainous, A.G. and Kohrs, F.P. (1995) A Comparison of Health Status between Rural and Urban Adults. Journal of Community Health, 20, 423-431.

https://doi.org/10.1007/BF02260438

[12] Friedman, B., Conwell, Y. and Delavan, R.L. (2007) Correlates of Late-Life Major 
Depression: A Comparison of Urban and Rural Primary Care Patients. The American Journal of Geriatric Psychiatry, 15, 28-41.

https://doi.org/10.1097/01.JGP.0000224732.74767.ad

[13] Lee, C.J. (2009) A Comparison of Health Promotion Behaviors in Rural and Urban Community-Dwelling Spousal Caregivers. Journal of Gerontological Nursing, 35, 34-40. https://doi.org/10.3928/00989134-20090331-04

[14] Wang, H.H., Shieh, C. and Wang, R.H. (2004) Self-Care and Well-Being Model for Elderly Women: A Comparison of Rural and Urban Areas. Kaohsiung Journal of Medical Science, 20, 63-68. https://doi.org/10.1016/S1607-551X(09)70086-5

[15] Chiu, H.C., Chen, C.M., Huang, C.J. and Mau, L.W. (2005) Depressive Symptoms, Chronic Medical Conditions and Functional Status: A Comparison of Urban and Rural Elders in Taiwan. International Journal of Geriatric Psychiatry, 20, 635-644. https://doi.org/10.1002/gps.1292

[16] Apidechkul, T. (2011) Comparison of Quality of Life and Mental Health among Elderly People in Rural and Suburban Areas, Thailand. Southeast Asian Journal of Tropical Medicine and Public Health, 42, 1282-1292.

[17] Imaiso, J. (2015) Negative/Positive Home-Based Caregiving Appraisals by Informal Carers of the Elderly in Japan. Primary Health Care Research \& Development, 16 167-178. https://doi.org/10.1017/S1463423614000309 